\title{
Weakly nonlinear analysis of the electrohydrodynamic instability of a charged membrane
}

\author{
R. M. Thaokar and V. Kumaran \\ Department of Chemical Engineering, Indian Institute of Science, Bangalore 560 012, India
}

\begin{abstract}
The effect of nonlinear interactions on the linear instability of shape fluctuations of a flat charged membrane immersed in a fluid is analyzed using a weakly nonlinear stability analysis. There is a linear instability when the surface tension reduces below a critical value for a given charge density, because a displacement of the membrane surface causes a fluctuation in the counterion density at the surface, resulting in an additional Maxwell normal stress at the surface which is opposite in direction to the stress caused by surface tension. The nonlinear analysis shows that at low surface charge densities, the nonlinear interactions saturate the growth of perturbations resulting in a new steady state with a fluctuation amplitude determined by the balance between the destabilizing electrodynamic force and surface tension. As the surface charge density is increased, the nonlinear terms destabilize the perturbations, and the bifurcation is subcritical. There is also a significant difference in the predictions of the approximate Debye-Huckel and more exact Poisson-Boltzmann equations at high charge densities, with the former erroneously predicting that the bifurcation is supercritical at all charge densities.
\end{abstract}

\section{INTRODUCTION}

It is well known that adsorbed charges on membrane surfaces have a significant effect on the functioning of membranes in biological systems. There are significant variations in charge distribution [1] and the transmembrane potential when there are shape changes in membranes. There has also been experimental evidence to indicate that variation in charge densities could be important in influencing the shapes of vesicles made of lipid bilayers. Vesicles are usually made under nonequilibrium conditions, because the bending energy for the formation of a vesicle of micron size is large compared to the thermal energy. However, some interesting experimental results [2] have revealed that stable vesicles could be made at equilibrium if a mixture of lipids with surface charges of opposite signs are used. There have been many studies on shape changes due to the asymmetry of inclusions in the membrane and their phase separation on the surface, and other nonequilibrium processes [3]. Phase separation of the components of a membrane could also alter the shape $[4,5]$, but it is expected that effects such as head-tail asymmetry would lead to structures with characteristic lengths of the same magnitude as the domains on the surface, whereas typical sizes of vesicles could be two to three orders of magnitude larger than the membrane thickness. Since shape changes in biological membranes are accompanied by changes in the transmembrane potential, it is useful to examine whether shape changes of flexible charged surfaces could be caused by changes in the surface potential. The linear stability of surface fluctuations on a charged surface has been studied as a function of the surface potential [6], and it is known that surface fluctuations become unstable when the surface potential exceeds a critical value at a given surface tension, or when the surface tension reduces below a critical value for a given surface potential. However, the effect of nonlinear interactions on the growth of the fluctuations cannot be studied using a linear analysis. The objective of the present analysis is to examine whether nonlinear interactions saturate the linearly unstable shape fluctuations, leading to a supercritical state, or whether the bifurcation is subcritical where nonlinearities destabilize the linearly stable state.

It is well known that the presence of adsorbed charges could significantly alter the elasticity of membranes. Winterhalter and Helfrich [7] and Lekkerkerker [8], as well as the subsequent studies $[9,10]$, found that there is an increase in the elasticity due to adsorbed charges. There is a change in the modulus for the mean curvature and the Gaussian curvature due to adsorbed charges, and the change in the Gaussian curvature could favor the spontaneous formation of vesicles. In these studies, the change in the electrostatic energy due to the curvature of the membrane is determined, and the corrections to the elasticity moduli are calculated from the free energy change. The corrections to the elastic moduli are manifested as additional contributions to the curvature energy when a net curvature is imposed on the membrane. It has been shown [11] that a difference in the charge densities in the two lipid layers forming a bilayer could stabilize a vesicle, because there is a reduction in electrostatic energy when the higher charge density is on the outside of the vesicle. This could compensate for the increase in the curvature energy. The effect of charge density curvature coupling on the dynamics of fluctuations on a charged surface was analyzed [12]. The analysis showed that when the charges are permitted to move on the membrane surface, there is an instability of the flat state of the membrane due to a correlated variation in the charge density and the curvature. However, this analysis assumed that the thickness of the counterion layer at the surface is small compared to the wavelength of the perturbations, and variations in the counterion density parallel to the surface were neglected.

In the present analysis, we carry out a weakly nonlinear analysis of the fluctuations at the surface of a charged membrane. The corresponding linear analysis [6] indicated that perturbations become unstable when the surface charge density exceeds a critical value for a given membrane tension, or the tension reduces below a critical value for a given surface 
charge density. However, the linear analysis cannot be used to determine the amplitude of fluctuations, and it is necessary to include higher order terms in the amplitude expansion. In the present case, a Landau analysis is used where the next higher (cubic) term in the amplitude expansion is included, and the "Landau constant," which is the coefficient of the cubic term in the amplitude expansion, is calculated. If the coefficient of the cubic term is negative, the system is supercritically stabilized by nonlinear interactions at a nonzero amplitude. If the coefficient of the cubic term is positive, the system is destabilized by nonlinear interactions. The analysis is carried out using the Poisson-Boltzmann equation for the relation between the charge density and potential, as well as the simpler Debye-Huckel approximation. The DebyeHuckel approximation is valid only at low charge densities, while the Poisson-Boltzmann equation is applicable to higher charge densities as well. One of the important results of the analysis is that there is a significant difference in the results of the nonlinear analysis for the two models even when the linear stability analysis provides results that are in good agreement.

It is important to note that the wavelength of perturbations in this case is of the same magnitude as the thickness of the counterion layer near the surface. The counterion layer thickness under physiological conditions is about $1 \mathrm{~nm}$, which is small compared to the length scale of structures such as vesicles. However, the linear stability analysis [6] predicts that the most unstable mode for a flat membrane has zero wave number, indicating that the most unstable mode for a system of finite size is likely to be the size of the system itself. However, the selection of the most unstable mode is likely to depend very sensitively on the surface potential when the size of the structure is large compared to the thickness of the counterion layer. There are other situations where the thickness of the counterion layer could increase to $1 \mu \mathrm{m}$ when the salt concentration is decreased, and the results of the present analysis would be directly applicable in those cases.

The linear stability analysis [6] showed that the inertial and convective terms in the momentum and concentration equations are zero for neutrally stable modes, and so it is assumed in the present case that the Reynolds and Peclet numbers are zero. The limit of low Reynolds number is appropriate for micron scale structures in biological systems. The validity of the zero Peclet number limit can be estimated as follows. The diffusion of a small molecule in a liquid is $O\left(10^{-9} \mathrm{~m}^{2} / \mathrm{s}\right)$, and the Peclet number $(U L / D)$ is small for structures of micron scales $L \sim 10^{-6} \mathrm{~m}$ if the velocity scale is smaller than $10^{-3} \mathrm{~m} / \mathrm{s}$. For membranes with surface tension and in the absence of fluid inertia, a characteristic velocity scale can be estimated as $(\Gamma / \mu)$, where $\Gamma$ is the surface tension and $\mu$ is the viscosity. The viscosity of water is $O\left(10^{-3} \mathrm{~kg} / \mathrm{m} \mathrm{s}\right)$, and therefore the velocity is small compared to $10^{-3} \mathrm{~m} / \mathrm{s}$ for $\Gamma<10^{-6} \mathrm{~kg} / \mathrm{m} \mathrm{s}^{2}$. This is about three orders of magnitude less than the surface tension of an airwater interface, and therefore the present analysis is likely to be applicable only for membranes with very low tension.

In this analysis, we assume that the charge densities on the two sides of the membrane are decoupled. This is valid when the dielectric constant of the hydrophobic tails in the lipid layer is small compared to the dielectric constant of the surrounding water. In practical situations, the ratio is about $1 / 40$, so the approximation is valid for distances about 40 times the bilayer thickness [7]. Though this is not strictly true in cases where the dielectric constants are comparable, we use this as a first approximation to make the problem analytically tractable.

\section{GOVERNING EQUATIONS}

A two-dimensional coordinate system is used for analyzing the perturbations, where $y^{*}$ is the direction normal to the membrane and $x^{*}$ is the direction in the plane of the membrane. The superscript "*” indicates dimensional quantities, while nondimensional quantities are written without a superscript. The membrane is flat in the base state, and is located at position $y^{*}=0$ separating two Newtonian fluids which extend to infinity in the $y^{*}$ direction. The membrane has a charge density $\sigma^{*}$, while the concentration of the charged species at a large distance from the membranes (where the solution is neutral) is $N_{\infty}$. In the vicinity of the membrane, there is a double layer with charge densities $n_{+}^{*}\left(y^{*}\right)$ and $n_{-}^{*}\left(y^{*}\right)$. The electrolyte is considered to be symmetric so that the number of charges per ion are equal for the two charged species, $z_{+}=z_{-}=z$.

The incompressible Navier-Stokes equations for the fluid, the concentration equations for the charged species, and the Poisson-Boltzmann equation relating the potential to the charge density are

$$
\begin{aligned}
& \nabla^{*} \cdot \mathbf{v}^{l *}=0, \\
& \rho^{*}\left(\partial_{t}^{*} \mathbf{v}^{l *}+\mathbf{v}^{l *} \cdot \nabla^{*} \mathbf{v}^{l *}\right)=-\boldsymbol{\nabla} * p_{f}^{l *}+\eta^{*} \nabla^{* 2} \mathbf{v}^{l *} \\
& +\left(n_{+}^{l *}-n_{-}^{l *}\right) e \mathbf{E}^{\mathbf{1} *}, \\
& \partial_{t} n_{+}^{l *}+\mathbf{v}^{l *} \cdot \boldsymbol{\nabla} n_{+}^{*}=\boldsymbol{\nabla}^{*} \cdot D\left[\boldsymbol{\nabla}^{*} n_{+}+\frac{z e n_{+}^{*}}{T} \boldsymbol{\nabla}^{*} \psi^{l *}\right], \\
& \partial_{t} n_{-}^{l *}+\mathbf{v}^{l *} \cdot \boldsymbol{\nabla} n_{-}^{*}=\nabla^{*} \cdot D\left[\nabla^{*} n_{-}-\frac{z e n_{-}^{*}}{T} \nabla^{*} \psi^{l *}\right], \\
& \nabla^{* 2} \psi^{l *}=-\frac{\rho_{c}^{l *}}{\epsilon} .
\end{aligned}
$$

where the superscript $l$ is used to distinguish between the fluid on the two sides of the membrane, $l=a$ for the fluid in the half-space $y^{*}>0$, and $b$ for fluid in the half-space $y^{*}$ $<0$ in the base state; $\mathbf{v}^{*}$ and $p_{f}^{*}$ are the velocity and pressure in the fluid, $\rho^{*}$ and $\eta^{*}$ are the density and viscosity which are assumed to be equal for the two fluids for simplicity, $e$ is the charge of an electron, $\mathbf{E}^{*}=-\nabla^{*} \psi^{*}$ is the electric field, $\psi^{*}$ is the scalar electric potential field, and $\partial_{t}^{*} \equiv\left(\partial / \partial t^{*}\right)$.

We consider perturbations with wavelength of the same magnitude as the Debye length, so that the following scalings are used- $\psi^{*}=T /(z e) \psi, \quad \mathbf{x}^{*}=\kappa^{-1} \mathbf{x}, \quad \mathbf{v}^{*}$ $=\left[N_{\infty} T /(\eta \kappa) \mathbf{v}\right], \quad p_{f}^{*}=N_{\infty} T p_{f}$, and time $t^{*}=\eta /\left(N_{\infty} T\right) t$, 
where $\kappa=\left(N_{\infty} z^{2} e^{2} /(\epsilon T)\right)^{1 / 2}$ is the inverse of Debye screening length. With these scalings, the equations become

$$
\begin{gathered}
\boldsymbol{\nabla} \cdot \mathbf{v}^{l}=0, \\
\operatorname{Re}\left(\partial_{t} \mathbf{v}^{l}+\mathbf{v}^{l} \cdot \boldsymbol{\nabla}\left(\mathbf{v}^{l}\right)\right)=-\boldsymbol{\nabla} p_{f}^{l}+\nabla^{2} \mathbf{v}^{l}+\left(\nabla^{2} \psi^{l}\right) \boldsymbol{\nabla} \psi^{l}, \\
\operatorname{Pe}\left(\partial_{t} n_{+}^{l}+\mathbf{v}^{l} \cdot \boldsymbol{\nabla} n_{+}\right)=\boldsymbol{\nabla} \cdot\left[\boldsymbol{\nabla} n_{+}+n_{+} \boldsymbol{\nabla} \psi^{l}\right], \\
\operatorname{Pe}\left(\partial_{t} n_{-}^{l}+\mathbf{v}^{l} \cdot \boldsymbol{\nabla} n_{-}\right)=\boldsymbol{\nabla}\left[\boldsymbol{\nabla} n_{-}-n_{-} \boldsymbol{\nabla} \psi^{l}\right] . \\
\boldsymbol{\nabla}^{2} \psi^{l}=-\left(n_{+}-n_{-}\right)
\end{gathered}
$$

where $\operatorname{Re}=\left(\rho N_{\infty} T\right) /\left(\eta^{2} \kappa^{2}\right)$ is the Reynolds number (ratio of inertial and viscous forces for the velocity fluctuations) and $\mathrm{Pe}=N_{\infty} T /\left(\eta D \kappa^{2}\right)$ is the Peclet number (ratio of convective and diffusive effects for the electrolyte concentration field). The fluid mass and momentum equations are considerably simplified for the case $\mathrm{Re}=0$ and $\mathrm{Pe}=0$

$$
\begin{gathered}
\boldsymbol{\nabla} \cdot \mathbf{v}^{l}=0, \\
-\boldsymbol{\nabla} p_{f}^{l}+\nabla^{2} \mathbf{v}^{l}+\left(\boldsymbol{\nabla}^{2} \psi^{l}\right) \boldsymbol{\nabla} \psi^{l}=0,
\end{gathered}
$$

while the Poisson-Boltzmann equation for the potential can be written as

$$
\boldsymbol{\nabla}^{2} \psi^{l}=\sinh \left[\psi^{l}\right] .
$$

For small potentials, $\left(z e \psi^{*} / T\right) \ll 1$, the Poisson-Boltzmann equation reduces to a linear equation (Debye-Huckel approximation)

$$
\nabla^{2} \psi^{l}=\psi^{l} .
$$

In the present study, detailed analytical results are provided for the Debye-Huckel approximation (henceforth called the DH model), while numerical results of the nonlinear Poisson-Boltzmann equation (PB model) are also provided. The details of the numerical procedure are given in the Appendix B.

In the base state, the pressure and electrical potential vary only in the $y$ direction, and the governing equations are

$$
\begin{gathered}
-d_{y} P_{f}^{l}+\left(d_{y}^{2} \Psi^{l}\right)\left(d_{y} \Psi^{l}\right)=0, \\
d_{y}^{2} \Psi^{l}=F_{1}\left[\Psi^{l}\right],
\end{gathered}
$$

where $d_{y} \equiv(d / d y), \quad F_{1}\left[\Psi^{l}\right]=\sinh \left[\Psi^{l}\right]$, and $\Psi^{l}$ for the PB and $\mathrm{DH}$ models respectively. The solutions for the potentials in the base state are

$$
\begin{aligned}
& \Psi^{a}=4 \tanh ^{-1}\left(e^{-y} \tanh \left[\frac{\Psi_{s}^{a}}{4}\right]\right), \\
& \Psi^{b}=4 \tanh ^{-1}\left(e^{y} \tanh \left[\frac{\Psi_{s}^{b}}{4}\right]\right) .
\end{aligned}
$$

The following simplified solutions are obtained using the Debye-Huckel approximation:

$$
\begin{gathered}
\Psi^{a}=\Psi_{s}^{a} \exp (-y), \\
\Psi^{b}=\Psi_{s}^{b} \exp (y) .
\end{gathered}
$$

The mean fluid pressure is related to the potential by

$$
P_{f}^{l}=\frac{1}{2}\left(\frac{\partial \Psi^{l}}{\partial y}\right)^{2}
$$

\section{A. Boundary conditions}

Since the membrane surface fluctuates, boundary conditions are applied on the boundary that varies with position. We consider the potential at the surface of the membrane to be fixed in the present analysis, and the boundary condition for the surface potential is

$$
\left.\psi^{l}\right|_{x, \eta}=\boldsymbol{\Psi}_{m}^{l},
$$

where $\left.\psi^{l}\right|_{x, \eta}$ is the potential at the perturbed interface while $\boldsymbol{\Psi}_{m}^{l}$ is the mean surface potential. The boundary condition for the fluid velocity and stress fields are as follows. In the limit where the amplitude of perturbations is large compared to the thickness of the membrane, the tangential velocity at the surface is small compared to the normal velocity, and so the tangential velocity can be assumed to be zero in the leading approximation. The normal velocity of the fluid at the surface of the membrane is equal to the velocity of the membrane in the normal direction, while the difference between the normal fluid stresses is balanced by the normal force due to surface tension.

While implementing boundary conditions of the type (22), the interface position is not known a priori, but is determined as a part of solution, and so some care has to be taken while applying boundary conditions. Consider a material point on the unperturbed membrane which is labeled by its $x$ coordinate, $x$. After deformation, this moves to a new position $\eta(x)$, where $\eta(x)$ is the vertical component of the $L a$ grangian displacement of the material point at the interface. By definition, the components of the Eulerian displacement field $\mathbf{u}$ are given by

$$
u_{y}(x, t)=\eta
$$

The unit normal $\mathbf{n}$ and the unit tangent $\mathbf{t}$ to the perturbed interface are defined as

$$
\mathbf{n}=\frac{-\mathbf{e}_{\mathbf{x}}\left(\frac{\partial \eta}{\partial x}\right)+\mathbf{e}_{\mathbf{y}}}{\sqrt{1+\left(\frac{\partial \eta}{\partial x}\right)^{2}}} \mathbf{t}=\frac{\mathbf{e}_{x}+\mathbf{e}_{y}\left(\frac{\partial \eta}{\partial x}\right)}{\sqrt{1+\left(\frac{\partial \eta}{\partial x}\right)^{2}}} .
$$

The matching conditions for the velocity at the perturbed interface $(x, \eta)$ are

$$
\begin{gathered}
\left.\left(\mathbf{n} \cdot \mathbf{v}^{a}\right)\right|_{x, \eta}=\left.\left(\mathbf{n} \cdot \mathbf{v}^{b}\right)\right|_{x, \eta}=\left.\left(\mathbf{n} \cdot \mathbf{v}^{m}\right)\right|_{x, \eta}, \\
\left.\left(\mathbf{t} \cdot \mathbf{v}^{a}\right)\right|_{x, \eta}=\left.\left(\mathbf{t} \cdot \mathbf{v}^{b}\right)\right|_{x, \eta}=0,
\end{gathered}
$$


where the superscript $m$ refers to variables defined on the membrane surface. The scaled normal stress balance condition is

$$
[\mathbf{n} \cdot \boldsymbol{\tau} \cdot \mathbf{n}]_{x, \eta}^{a}-[\mathbf{n} \cdot \boldsymbol{\tau} \cdot \mathbf{n}]_{x, \eta}^{b}=\Gamma\left(\boldsymbol{\nabla}_{s} \cdot \mathbf{n}\right)_{x, \eta},
$$

where $\boldsymbol{\nabla}_{s}$ is the surface gradient along the membrane surface, given by $\boldsymbol{\nabla}_{s}=[\boldsymbol{\nabla}-\mathbf{n}(\mathbf{n} \cdot \boldsymbol{\nabla})]$, where $\boldsymbol{\nabla}$ is the threedimensional gradient operator, and $\boldsymbol{\sigma}$ is the stress tensor along the membrane. The term $\boldsymbol{\nabla}_{s} \cdot \mathbf{n}$ is the negative of the mean curvature of the membrane surface, and the term proportional to this accounts for the normal stress exerted due to surface tension.

The terms in the boundary conditions (25)-(27) are expanded in a Taylor series in the parameter $\eta=u_{y}$.

$$
\begin{aligned}
& \left.\left(\mathbf{t} \cdot \mathbf{v}^{l}\right)\right|_{x, \eta}=\left[v_{x}^{l}+v_{y}^{l}\left(\frac{\partial u_{y}}{\partial x}\right)\right]\left[1-\frac{1}{2}\left(\frac{\partial u_{y}}{\partial x}\right)^{2}+\cdots\right], \\
& \left.\left(\mathbf{n} \cdot \mathbf{v}^{l}\right)\right|_{x, \eta}=\left[v_{y}^{l}-v_{x}^{l}\left(\frac{\partial u_{y}}{\partial x}\right)\right]\left[1-\frac{1}{2}\left(\frac{\partial u_{y}}{\partial x}\right)^{2}+\cdots\right],
\end{aligned}
$$

$$
=\left.\frac{\left.\left[\left(\tau_{x x}^{l} \frac{\partial u_{y}}{\partial x}-\tau_{x y}^{l}\right)\left(\frac{\partial u_{y}}{\partial x}\right)+\left\{-\tau_{x y}^{l}\left(\frac{\partial u_{y}}{\partial x}\right)+\tau_{y y}^{l}\right\}\right)\right]}{1+\left(\frac{\partial u_{y}}{\partial x}\right)^{2}}\right|_{x, \eta}
$$

$$
\left.\boldsymbol{\nabla}_{s} \cdot \mathbf{n}\right|_{x, \eta}=-\left.\frac{\left(\frac{\partial^{2} u_{y}}{\partial x^{2}}\right)}{\left[1+\left(\frac{\partial u_{y}}{\partial x}\right)^{2}\right]^{3 / 2}}\right|_{x, \eta}
$$

where the constitutive relation for the fluid stresses is

$$
\tau=-p_{f} \mathbf{I}+\left[\boldsymbol{\nabla} v+(\boldsymbol{\nabla} v)^{T}\right]+\boldsymbol{\nabla} \psi \boldsymbol{\nabla} \psi-\frac{1}{2} \mathbf{I}(\boldsymbol{\nabla} \psi) \cdot(\boldsymbol{\nabla} \psi) .
$$

Since the position of the interface has to be determined as a part of the solution, the boundary conditions at the perturbed interface are expanded about their values at the unperturbed interface $y=0$. If $F$ indicates a fluid parameter (fluid velocity, stresses), then $\left.F\right|_{x, \eta}$ at the perturbed interface are expanded in a Taylor series about their values at $(x, 0)$

$$
\left.F\right|_{x, \eta}=[F]_{0}+\left[\partial_{y} F\right]_{0} \eta+\frac{1}{2}\left[\partial_{y}^{2} F\right]_{0} \eta^{2}+\cdots
$$

where $[\cdots]_{0}$ represent quantities evaluated at the unperturbed interface, and $\eta$ is obtained as a part of the solution. From the above expressions, infinite series representations for the flow quantities are obtained as functions of $\eta$ and these are truncated at the required order in the weakly nonlinear theory. The Eulerian velocity field in the membrane $\left(v_{i}^{m}\right)$ is defined as the substantial derivative of the displacement field

$$
v_{y}^{m}=D_{t} u_{y},
$$

where $D_{t}=\partial_{t}+\mathbf{v} \cdot \boldsymbol{\nabla}$ is the substantial derivative.

\section{STABILITY ANALYSIS}

Linear stability studies [6] have indicated that perturbations become unstable when the surface potential exceeds a critical value for a given surface tension, or when the surface tension is decreased below a critical value for a given surface potential. However, the linear growth of perturbations is affected by nonlinearities both in governing equations as well as in the boundary conditions, although the nonlinearities in the governing equations are not present in the Debye-Huckel approximation. The nonlinearities in the boundary conditions arise due to Taylor expansion of the boundary conditions about the unperturbed state, as well as due to the variation of the surface normal along the perturbed interface. The linear stability analysis (discussed in Appendix A) indicates that perturbations become unstable in the zero wave number limit $k \rightarrow 0$. However, in real systems there is a minimum permissible wave number of perturbations due to the finite lateral extent of the system. Perturbations with this lowest permissible wave number become unstable first when the surface potential exceeds the critical value, and the effect of nonlinearities on the growth of these perturbations is analyzed in the weakly nonlinear analysis. In the weakly nonlinear theory, we aim to find the state of the system after it is rendered linearly unstable. The system is therefore assumed to have a tension $\Gamma$ slightly smaller than the critical tension $\Gamma_{c}$ given by the linear theory. When $\Gamma$ is slightly smaller than $\Gamma_{c}$, perturbations with wave number $k_{c}$ and lower become unstable and generate higher harmonics due to nonlinear interactions. It is useful to define the function $E(x)$ $=\exp \left[i\left(k_{c} x+\omega_{c} t\right)\right]$. In the weakly nonlinear theory, an expansion is used in the harmonic series as well as the amplitude of the perturbations

$$
\phi(x, y, t)=\sum_{s=0}^{\infty} \sum_{n=s}^{\infty}\left[A_{1}(\tau)\right]^{n}\left[E^{s} \widetilde{\phi}^{(s, n)}(y)+E^{-s} \widetilde{\phi}^{\dagger(s, n)}(y)\right],
$$

where the integer superscript $s$ indicates the harmonics with wave number $\left(s k_{c}\right)$ and frequency $\left(s \omega_{c}\right)$, the integer superscript $n$ indicates the order in powers of the amplitude of the perturbation, the superscript ${ }^{\dagger}$ is the complex conjugate, $A_{1}(\tau)=\epsilon A(\tau)$ is the amplitude of the wave that varies in the slow time scale $\tau$ (to be defined below), $\epsilon$ is a small parameter defined later, and $A(\tau)$ is an $O(1)$ quantity. It should be noted that $A_{1}(\tau)$ and $A(\tau)$ are real. As an aside, it is possible at this stage to let the amplitude $A$ vary as a function of a slow spatial variable in the $x$ direction. This would result in an envelope equation (a partial differential equation) for $A$ as a function of the slow spatial and temporal variables. In the expansion (35), $\widetilde{\phi}^{(0,0)}$ refers to the variables in the mean flow, while $\widetilde{\phi}^{(1,1)}$ are the perturbations in the linear stability analysis, and the results of the linear stability analysis are obtained by truncating the expansion (35) at $s=1, n=1$. To determine whether the linear instability is supercritical or 
subcritical, it is also necessary to consider equations for the perturbations at order $s=0, n=2, s=2, n=2$, and $s=1, n$ $=3$, as shown in Appendix A.

The slow time scale $\tau$ referred to below Eq. (35) arises for the following reason. In the vicinity of the transition point $\left(\Gamma_{c}, k_{c}\right)$, the amplitude is governed by the Landau expansion

$$
A_{1}(\tau)^{-1} d_{t} A_{1}(\tau)=s_{r}^{(0)}+A_{1}(\tau)^{2} s_{r}^{(1)}+\cdots,
$$

where $s_{r}^{(0)}$ is the real part of the linear growth rate $s^{(0)}$ and $s_{r}^{(1)}$ is the real part of the first Landau constant $s^{(1)}$. Near the linear neutral curve, $s_{r}^{(0)} \sim\left(\Gamma-\Gamma_{c}\right)$, and $s_{r}^{(0)}$ can be written as $s_{r}^{(0)}=\left(d s_{r}^{(0)} / d \Gamma\right)_{c}\left(\Gamma-\Gamma_{c}\right)$. If $s_{r}^{(1)}$ is $O(1)$, then the second term in the right-hand side of Eq. (36) is $O\left(\epsilon^{2}\right)$, and a balance is achieved if $\left(\Gamma-\Gamma_{c}\right)\left(d s_{r}^{(0)} / d \Gamma\right)_{c} \sim \epsilon^{2}$. For definiteness, let $\left(\Gamma-\Gamma_{c}\right)=\Gamma_{2} \epsilon^{2}$, where $\Gamma_{2}$ is an $O(1)$ quantity whose sign determines whether we are in the stable or unstable region around the neutral curve (a negative $\Gamma_{2}$ is in the unstable region). Now, this term should be balanced by the term on the left-hand side of Eq. (36), and so we introduce the slow time scale in the time derivative as $d_{t}=\epsilon^{2} d_{\tau}$. Since $A_{1}(\tau)$ is independent of the fast time scale $t$, the above equation becomes

$$
A^{-1} d_{\tau} A=\Gamma_{2}\left(d s_{r}^{(0)} / d \Gamma\right)_{c}+s_{r}^{(1)} A^{2} .
$$

This is the "scaled" version of the Landau equation in the vicinity of the critical point of the linear neutral curve. The objective of the rest of the analysis is to determine $s_{r}^{(1)}$ which determines whether the instability is subcritical or supercritical.

The details of the weakly nonlinear analysis are given in Appendix A. The boundary conditions for the problem at order $(s, n)$ contain inhomogeneous terms of order $(s, m)$ where $m<n$. Thus, the original nonlinear problem with an unknown membrane interface is reduced to a hierarchy of linear (but inhomogeneous) problems, which are solved beginning from the linear $(1,1)$ problem. The nonlinear analysis was carried out both for the PB as well as DH models. The linear stability analysis shows that the value of critical surface tension for both the Poisson-Boltzmann and the Debye-Huckel models is of the same order for $\Psi_{s}^{a} \sim O(1)$. However the nonlinear analysis can be quite different even for the regime of $\Psi_{s}^{a} \sim O(1)$ where the linear stability results agree. This is because the governing equations in the case of the DH model are linear at all orders $n$, while they are are nonlinear for the PB model and these nonlinearities can affect the results considerably.

\section{RESULTS}

The linear stability analysis for $s=1, n=1$ (Appendix A) shows that a system is linearly unstable when the scaled surface tension $\chi=\Gamma /\left(\left(\Psi_{s}^{a}\right)^{2}+\left(\Psi_{s}^{b}\right)^{2}\right)$ decreases below a critical value. Figure 1 shows the linear neutral stability curve for the system for different values of the surface potential; the region above the curve is stable while that below the curve is unstable. The neutral stability curve shows that the scaled value of the critical surface tension asymptotes to

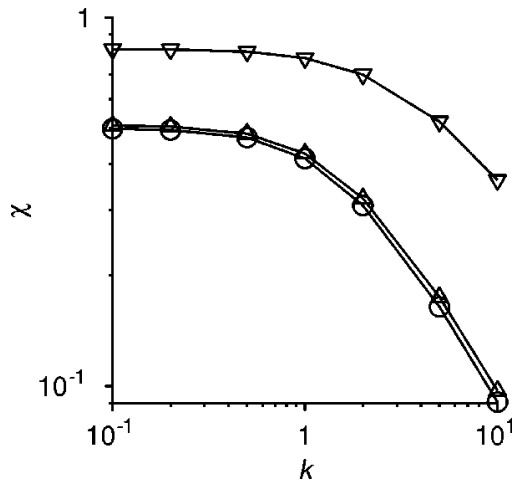

FIG. 1. Linear neutral stability curve for the charged membrane, a comparison of the $\mathrm{DH}$ and $\mathrm{PB}$ models for $\Psi_{s}^{b}=\Psi_{s}^{a}$. $\bigcirc, \mathrm{DH}$ model; $\triangle$, PB model $\Psi_{s}^{a}=1.0 ; \nabla$, PB model $\Psi_{s}^{a}=5.0$.

a constant value in the low wave number regime as can be seen from the analytical expression (A19). However, for wave numbers $k \gg 1$ the critical surface tension scales as $k^{-1}$. The critical surface tension as predicted by models DH and $\mathrm{PB}$ agree well for low values of surface potentials, but the DH model underestimates the critical surface tension for larger potentials. This trend can be easily understood, since the gradients of mean potential, as obtained from the PB model, are higher than that by the DH model, thereby rendering the system more unstable.

The Landau equation can be derived in the $k \rightarrow 0$ limit for the case of the DH model

$$
\frac{1}{A(\tau)} \frac{d A(\tau)}{d \tau}=-\frac{k \Gamma_{2}}{4}+\frac{A^{2} k\left(-4+k^{2}\right)\left(1+r^{2}\right) \boldsymbol{\Psi}_{s}^{a 2}}{64} A(\tau)^{2} .
$$

The Landau coefficient is negative in the $k \rightarrow 0$ limit (note that $\Gamma_{2}$ is negative). Thus, the system is supercritically stable with an equilibrium amplitude given by

$$
A(\tau)=\sqrt{\frac{16\left|\Gamma_{2}\right|}{A^{2}\left(4-k^{2}\right)\left(1+r^{2}\right) \boldsymbol{\Psi}_{s}^{a 2}}},
$$

where $A(\tau)$ is the equilibrium amplitude while $A$ is the amplitude of height perturbations used for normalization and has been set equal to 1.0 in the present analysis.

For $k$ of $O(1)$, the reduced equilibrium amplitude

$$
A_{e q}=A(\tau) \sqrt{\left(\frac{\left(\Psi_{s}^{a}\right)^{2}+\left(\Psi_{s}^{b}\right)^{2}}{\Gamma_{2}}\right)}
$$

shows a maximum (Fig. 2) at finite wave number for the DH model. It is useful to compare the equilibrium amplitude obtained from the DH model with the PB model for $\Psi_{s}^{a}$ of $O$ (1) (Fig. 2). The results for the two models agree well for low surface potentials, but for surface potential of $O(1)$, there is significant difference between the equilibrium amplitudes of the two models, although the linear stability results compare well for $O(1)$ values of surface potential. This can be attributed to the nonlinearities present at each order which can lead to significantly different results. Although the DH 




FIG. 2. Variation of the reduced equilibrium amplitude with wave number $k$ for the PB and DH model for $\Psi_{s}^{b}=\Psi_{s}^{a}$. $\bigcirc, \mathrm{DH}$ model; $\triangle$, PB model $\Psi_{s}=0.1 ; \diamond$, PB model $\Psi_{s}=1.0 ; \nabla$, PB model $\Psi_{s}=3.0 ; \times$, PB model $\Psi_{s}=5.0$.

model indicates the presence of a supercritically stable state for all values of surface potentials, the PB model shows that the system goes from a supercritically stable state to a subcritical instability at large values of the surface potential. Table I gives the variation of the reduced Landau constant with surface potential at two different values of wave number $k$. The DH model predicts a constant value of the reduced Landau constant as can be seen from Eq. (38) and is negative, indicating supercritical stability. The PB model however, shows a bifurcation from supercritical stability to subcritical instability as the surface potential is increased and the Landau constant becomes positive at high values of surface potential.

This is more clearly seen in Figs. 3 and 4, where the reduced equilibrium amplitude is plotted as a function of the surface potential for two different wave numbers. The figures show that the equilibrium amplitudes predicted by the PB model are much lower than that for the DH model. However the PB model indicates a change to subcritical instability when $\Psi_{s}^{a}$ is $O(1)$ and this is indicated by a discontinuity in the curves.

\section{CONCLUSIONS}

The nonlinear analysis of the stability of fluctuations at a charged surface have revealed two important results.

(1) At low surface charge densities, the linear instability is

TABLE I. Variation of scaled Landau coefficient $-s_{r}^{(1)} /\left[\left(\Psi_{s}^{(a)}\right)^{2}+\left(\Psi_{s}^{(b)}\right)^{2}\right]$ with surface potential.

\begin{tabular}{lcccc}
\hline \hline & \multicolumn{2}{c}{$k=0.01$} & \multicolumn{2}{c}{$k=1.0$} \\
$\Psi_{s}^{a}$ & $\mathrm{DH}$ & $\mathrm{PB}$ & $\mathrm{DH}$ & $\mathrm{PB}$ \\
\hline 0.001 & $6.312 \times 10^{-4}$ & $6.168 \times 10^{-4}$ & $3.675 \times 10^{-2}$ & $3.675 \times 10^{-2}$ \\
0.01 & $6.312 \times 10^{-4}$ & $8.004 \times 10^{-4}$ & $3.675 \times 10^{-2}$ & $3.676 \times 10^{-2}$ \\
0.1 & $6.312 \times 10^{-4}$ & $2.900 \times 10^{-2}$ & $3.735 \times 10^{-2}$ & $3.676 \times 10^{-2}$ \\
1.00 & $6.312 \times 10^{-4}$ & 1.923 & $3.675 \times 10^{-2}$ & $7.133 \times 10^{-2}$ \\
3.00 & $6.312 \times 10^{-4}$ & -22.556 & $3.675 \times 10^{-2}$ & $-7.044 \times 10^{-1}$ \\
5.00 & $6.312 \times 10^{-4}$ & -952.325 & $3.675 \times 10^{-2}$ & -21.7484 \\
10.0 & $6.312 \times 10^{-4}$ & $-6.072 \times 10^{6}$ & $3.675 \times 10^{-2}$ & -133068 \\
\hline \hline
\end{tabular}

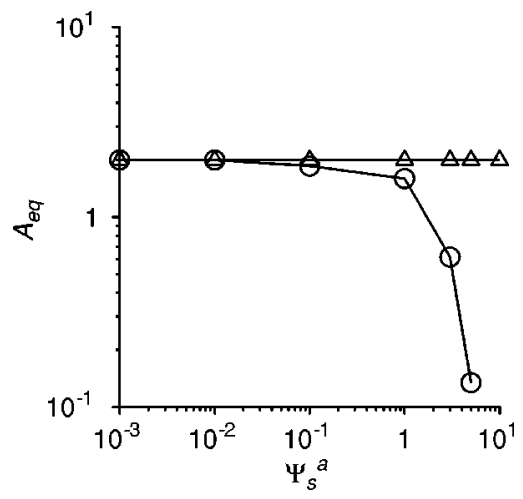

FIG. 3. Comparison of variation of equilibrium amplitude with the base state potential for different potentials for the PB and DH models for $\left(\Psi_{s}^{b}=\Psi_{s}^{a}\right)$ and $k=0.01 . \triangle$, DH model; $\bigcirc$, PB model.

stabilized by nonlinear interactions, and has a supercritical equilibrium state. The amplitude of fluctuations scales as $\left(\chi-\chi_{c}\right)^{1 / 2}$, where $\chi=\left(\Gamma /\left[\left(\Psi_{s}^{a}\right)^{2}+\Psi_{s}^{b}\right]^{2}\right)$ is the ratio of the surface tension and the square of the surface potential. The critical value $\chi_{c}$ approaches a value in the low wave number limit, and so the amplitude of perturbations is determined by a dynamical balance between the stabilizing surface tension and destabilizing surface potential. Therefore, it is expected that the amplitudes of long wave surface fluctuations are not determined from thermodynamic equipartition of energy considerations, but rather by electrodynamic considerations when the system is in the unstable regime for low surface charge densities. In this regime, the results of the DebyeHuckel and Poisson-Boltzmann equations are in good agreement.

(2) At higher surface potential, the Debye-Huckel approximation predicts that the system is still supercritically stable, but the Poisson-Boltzmann equation indicates that there is a subcritical bifurcation by which a linearly stable system is rendered unstable sufficiently close to the neutral stability curve. Thus, the system does not saturate to a new steady state at high surface potential, but has to undergo a shape change. The present analysis indicates that there is a

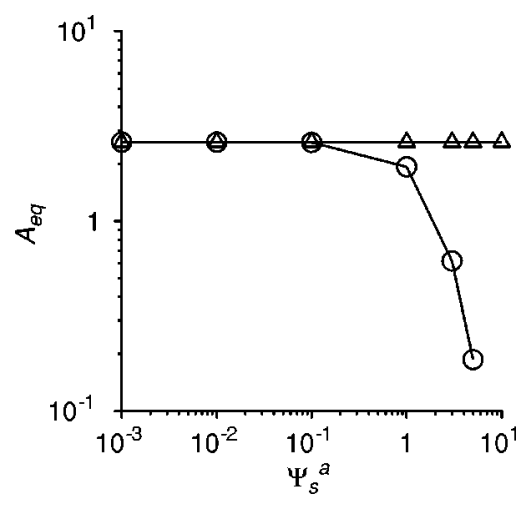

FIG. 4. Comparison of variation of the equilibrium amplitude with the base state potential for different potentials for the PB and $\mathrm{DH}$ models for $\left(\Psi_{s}^{b}=\Psi_{s}^{a}\right)$ and $k=1.0 . \triangle$, DH model; $\bigcirc$, PB model. 
qualitative difference in the predictions of the nonlinear analysis for the Debye-Huckel and Poisson-Boltzmann equations for $\chi \sim 1$, even when the predictions of the linear stability analysis are in close agreement. Thus, it is necessary to use the Poisson-Boltzmann equation to accurately capture the effect of nonlinearities on the growth of perturbations.

An issue of interest is whether the agreement can be obtained between the PB and DH models by renormalizing the surface potential in the DH model. This can be accomplished only when the qualitative nature of the bifurcation (supercritical or subcritical) is identical for the two models. The results of the present analysis indicate that at low surface potentials, the bifurcation is supercritical for both the DH and PB models, and so agreement can be obtained by renormalizing the potential in the DH model. However, at high potentials (for $\Psi_{a}^{s}$ greater than about 5, as shown in Figs. 3 and 4), the bifurcation is predicted to be supercritical by the DH model, but subcritical by the PB model. In this case, it is clear that agreement cannot be obtained by renormalizing the surface potential in the PB model.

\section{APPENDIX A}

The $s=1, n=1$ problem. The governing equations in the Fourier modes are

$$
\begin{gathered}
i k \tilde{v}_{x}^{l(1,1)}+\partial_{y} \tilde{v}_{y}^{l(1,1)}=0 \\
-i k \tilde{p}_{f}^{l(1,1)}+\left(\partial_{y}^{2}-k^{2}\right) \tilde{v}_{x}^{l(1,1)}+i k \psi^{l(1,1)}\left(\partial_{y}^{2} \boldsymbol{\Psi}^{l}\right)=0, \\
-\partial_{y} \tilde{p}_{f}^{l(1,1)}+\left(\partial_{y}^{2}-k^{2}\right) \tilde{v}_{y}^{l(1,1)}+\left(\partial_{y} \boldsymbol{\Psi}^{l}\right)\left(\partial_{y}^{2}-k^{2}\right) \psi^{l(1,1)} \\
+\left(\partial_{y} \psi^{l(1,1)}\right)\left(\partial_{y}^{2} \boldsymbol{\Psi}^{l}\right)=0 .
\end{gathered}
$$

These can be reduced to get a single governing equation for the $y$ velocity

$$
\begin{gathered}
\left(\partial_{y}^{2}-k^{2}\right) \widetilde{\psi}^{l(1,1)}=F_{2}\left[\widetilde{\psi}^{l(1,1)}\right], \\
\left(\partial_{y}^{2}-k^{2}\right)^{2} \widetilde{v}_{y}^{l(1,1)} 0,
\end{gathered}
$$

where $F_{2}\left[\widetilde{\psi}^{l(1,1)}\right]=\widetilde{\psi}^{l(1,1)} \cosh \left[\Psi^{l}\right]$ and $\widetilde{\psi}^{l(1,1)}$ for models PB and DH respectively. The resulting governing equations for the PB model have to be solved numerically, but analytical solutions can be obtained for the DH model

$$
\begin{gathered}
\widetilde{\psi}^{a(1,1)}=A_{3} \exp \left[-\sqrt{1+k^{2}} y\right], \\
\widetilde{\psi}^{b(1,1)}=B_{3} \exp \left[\sqrt{1+k^{2}} y\right], \\
\tilde{v}_{y}^{a(1,1)}=\left(A_{1}+A_{2} y\right) \exp [-k y], \\
\tilde{v}_{y}^{b(1,1)}=\left(B_{1}+B_{2} y\right) \exp [k y] .
\end{gathered}
$$

The boundary conditions are

$$
\tilde{v}_{y}^{a(1,1)}=\tilde{v}_{y}^{b(1,1)},
$$

$$
\begin{gathered}
\tilde{v}_{x}^{a(1,1)}+\left(\partial_{y} \mathbf{v}_{x}^{a(1,1)}\right) \tilde{u}_{y}^{(1,1)}=0, \\
\tilde{v}_{x}^{a(1,1)}+\left(\partial_{y} \mathbf{v}_{x}^{a(1,1)}\right) \tilde{u}_{y}^{(1,1)}=0, \\
\tilde{\tau}_{y y}^{a(1,1)}-\tilde{\tau}_{y y}^{b(1,1)}=\Gamma k^{2} \tilde{u}_{y}^{(1,1)}, \\
\psi^{a(1,1)}+\left(\partial_{y} \Psi^{a}\right) \tilde{u}_{y}^{(1,1)}=0, \\
\psi^{b(1,1)}+\left(\partial_{y} \Psi^{b}\right) \tilde{u}_{y}^{(1,1)}=0, \\
\tilde{v}_{y}^{a(1,1)}=s \tilde{u}_{y}^{(1,1)},
\end{gathered}
$$

where the normal stress perturbation is

$$
\tilde{\tau}_{y y}^{l(1,1)}=-\tilde{p}_{f}^{a(1,1)}+2 \partial_{y} \tilde{v}_{y}^{l(1,1)}+\left(\partial_{y} \tilde{\psi}^{l(1,1)}\right)\left(\partial_{y} \Psi^{l}\right)
$$

and the pressure is calculated from the $x$ direction momentum balance. The eigenfunctions are then substituted in the boundary conditions, and the resultant dispersion matrix is solved to obtain the eigenvalue $s$,

$$
s=-\frac{\Gamma k}{4}+\frac{\left(-1+\sqrt{1+k^{2}}\right)\left(1+r^{2}\right) \Psi_{s}^{a 2}}{4 k},
$$

where $r$ is the ratio of surface potentials $\left(\mathbf{\Psi}_{s}^{b}\right) /\left(\mathbf{\Psi}_{s}^{a}\right)$. The expression for the growth rate indicates that the Maxwell stress destabilizes long wavelength perturbations when the potentials increase beyond a critical value $\left(1+r^{2}\right) \Psi_{s}^{a}>2 \Gamma$, and wavelengths $k^{2}<k_{c}^{2}$ are rendered unstable where

$$
k_{c}^{2}=\frac{4\left[\left(\Psi_{s}^{a 2}+\Psi_{s}^{b 2}\right)-2 \Gamma\right]}{\left(\Psi_{s}^{a 2}+\Psi_{s}^{b 2}\right)} .
$$

The growth rate however is independent of the sign of the surface potential as expected. The results of linear stability theory for the case of the PB model are obtained by numerically calculating the eigenfunctions. The results of the two models are in good agreement for $\Psi_{s}^{a} \ll 1$, but are not in agreement when $\Psi_{s}^{a} \sim O(1)$. It should also be noted that the growth rate is real, so that the instability results in standing waves.

The $s=0, n=2$ problem. This problem represents the $x$-independent correction to the mean flow due to nonlinear interactions. The governing equations are

$$
\begin{gathered}
\partial_{y} \tilde{v}_{y}^{l(0,2)}=0, \\
\partial_{y}^{2} \widetilde{v}_{x}^{l(0,2)}-i k \widetilde{\psi}^{* a(1,1)} \partial_{y}^{2} \widetilde{\psi}^{a(1,1)}+i k \widetilde{\psi}^{a(1,1)} \partial_{y}^{2} \widetilde{\psi}^{* a(1,1)}=0, \\
-k^{2}\left(\widetilde{\psi}^{* l(1,1)} \partial_{y} \widetilde{\psi}^{l(1,1)}+\widetilde{\psi}^{l(1,1)} \partial_{y} \widetilde{\psi}^{* l(1,1)}\right)-2 \partial_{y} \widetilde{p}_{f}^{l(0,2)}
\end{gathered}
$$

$$
+\left(\partial_{y} \widetilde{\psi}^{* a(1,1)} \partial_{y}^{2} \widetilde{\psi}^{l(1,1)}+\partial_{y} \widetilde{\psi}^{a(1,1)} \partial_{y}^{2} \widetilde{\psi}^{* l(1,1)}\right)+2 \partial_{y}^{2} \psi^{l(0,2)} \partial_{y} \Psi^{l}
$$




$$
\begin{gathered}
+2 \partial_{y} \widetilde{\psi}^{l(0,2)} \partial_{y}^{2} \Psi^{l}=0, \\
\partial_{y}^{2} \psi^{l(0,2)}=F_{m}\left[\psi^{l(0,2)}\right],
\end{gathered}
$$

where for the DH model

$$
F_{m}\left[\psi^{l(0,2)}\right]=\widetilde{\psi}^{l(0,2)}
$$

and for the PB model

$$
F_{m}\left[\psi^{l(0,2)}\right]=2 \cosh \left[\widetilde{\psi}_{m}^{l}\right] \widetilde{\psi}^{l(0,2)}+\left(\widetilde{\psi}^{l(1,1)}\right)^{2} \sinh \left[\widetilde{\psi}_{m}^{l}\right] .
$$

The eigenfunctions consistent with the above equations for the case of the DH model are

$$
\begin{gathered}
\tilde{v}_{y}^{l(0,2)}=0, \\
\tilde{p}_{f}^{l(0,2)}=0, \\
\widetilde{\psi}^{l(0,2)}=M_{a 1}^{(0,2)} \exp [-y], \\
\widetilde{\psi}^{l(0,2)}=M_{b 1}^{(0,2)} \exp [y], \\
\tilde{v}_{x}^{a(0,2)}=M_{a 2}^{(0,2)}, \\
\tilde{v}_{x}^{b(0,2)}=M_{b 2}^{(0,2)} .
\end{gathered}
$$

The boundary conditions for the problem are lengthy and are not provided here. The normal velocity continuity boundary condition is identically satisfied and so is the normal stress boundary condition so that the mean pressure developed is set to zero. The tangential velocity boundary condition and the potential boundary condition then determine the mean correction to the tangential velocity and the potential. Both these corrections are exponentially decaying functions. The normalizations evaluated from the boundary conditions are

$$
\begin{gathered}
M_{a 1}^{(0,2)}=\left[A^{2}\left(-1+2 \sqrt{1+k^{2}}\right) \Psi_{s}^{a}\right] / 2, \\
M_{b 1}^{(0,2)}=\left[A^{2}\left(-1+2 \sqrt{1+k^{2}}\right) \Psi_{s}^{a} r\right] / 2, \\
M_{a 2}^{(0,2)}=0, \\
M_{b 2}^{(0,2)}=0 .
\end{gathered}
$$

The $\alpha^{\prime}=2, n=2$ problem. This problem represents the nonlinear correction to the second harmonic of the linearly unstable wavenumber $k$. The governing equations at order $\alpha$ $=2, n=2$ are the following:

$$
\begin{gathered}
\partial_{y} \widetilde{v}_{y}^{l(2,2)}+2 i \alpha \tilde{v}_{x}^{l(2,2)}=0, \\
-2 i \alpha \tilde{p}_{f}^{l(2,2)}+\left(\partial_{y}^{2}-4 \alpha^{2}\right) \tilde{v}_{x}^{l(2,2)}-i k^{3}\left(\widetilde{\psi}^{l(1,1)}\right)^{2} \\
+i k \widetilde{\psi}^{l(1,1)} \partial_{y}^{2} \widetilde{\psi}^{l(1,1)} \\
+2 i k \widetilde{\psi}^{l(2,2)}\left(\partial_{y}^{2} \Psi_{s}^{a}\right)+2 i k \widetilde{\psi}^{l(2,2)} \partial_{y}^{2} \Psi_{s}^{l}=0,
\end{gathered}
$$

$$
\begin{gathered}
-\partial_{y} \tilde{p}_{f}^{l(2,2)}+\left(\partial_{y}^{2}-4 \alpha^{2}\right) \tilde{v}_{x}^{l(2,2)}-k^{2}\left(\widetilde{\psi}^{l(1,1)}\right) \partial_{y} \widetilde{\psi}^{l(1,1)} \\
-\partial_{y} \widetilde{\psi}^{l(1,1)} \partial_{y}^{2} \widetilde{\psi}^{l(1,1)} \\
+\left(\partial_{y}^{2}-4 \alpha^{2}\right)-\widetilde{v}_{y}^{l(2,2)}+\partial_{y} \widetilde{\psi}^{l(2,2)}\left(\partial_{y}^{2} \Psi_{s}^{a}\right)=0, \\
\left(\partial_{y}^{2}-4 \alpha^{2}\right) \widetilde{\psi}^{l(2,2)}=F_{3}\left[\widetilde{\psi}^{l(2,2)}\right],
\end{gathered}
$$

where for the DH model

$$
F_{2}\left[\widetilde{\psi}^{l(2,2)}\right]=\widetilde{\psi}^{l(2,2)}
$$

and for the PB model

$$
\begin{aligned}
F_{2}\left[\widetilde{\psi}^{l(2,2)}\right]= & 4 k^{2} \widetilde{\psi}^{l(2,2)}+\cosh \left[\Psi_{m}^{a} \widetilde{\psi}^{l(2,2)}\right] \\
& +\left\{\left(\widetilde{\psi}^{l(1,1)}\right)^{2} \sinh \left[\Psi_{s}^{m}\right]\right\} / 2 .
\end{aligned}
$$

The PB model is solved numerically. For the DH model however, analytical solutions are possible and we admit decaying solutions for this problem. The eigenfunctions consistent with the above governing equations can be written as

$$
\begin{gathered}
\widetilde{\psi}^{a(2,2)}=M_{a 1}^{(2,2)} \exp \left[-\sqrt{1+4 k^{2}} y\right], \\
\widetilde{\psi}^{b(2,2)}=M_{b 1}^{(2,2)} \exp \left[\sqrt{1+4 k^{2}} y\right], \\
\tilde{v}_{y}^{a(2,2)}=\frac{M_{a 2}^{(2,2)}+M_{a 3}^{(2,2)} y}{e^{2 k y}}, \\
\tilde{v}_{y}^{b(2,2)}=e^{2 k y}\left(M_{b 2}^{(2,2)}+M_{b 3}^{(2,2)} y\right) .
\end{gathered}
$$

At the interface $y=0$, there are seven inhomogeneous boundary conditions that can be used to calculate the eigenfunctions for the velocities and the potential. The constants can be easily evaluated as

$$
M_{a 2}^{(2,2)}=M_{a 3}^{(2,2)}=M_{b 2}^{(2,2)}=M_{b 3}^{(2,2)}=0
$$

$$
\begin{aligned}
& M_{a 1}^{(2,2)}=-\frac{A^{2} \boldsymbol{\Psi}_{s}^{a} F 1}{\left(-3+4 \sqrt{1+k^{2}}-\sqrt{1+4 k^{2}}\right)\left(1+r^{2}\right)}, \\
& M_{b 1}^{(2,2)}=-\frac{A^{2} r \mathbf{\Psi}_{s}^{a} F 2}{\left(-3+4 \sqrt{1+k^{2}}-\sqrt{1+4 k^{2}}\right)\left(1+r^{2}\right)},
\end{aligned}
$$




$$
\tilde{u}_{y}^{a(2,2)}=\frac{-\left\{A^{2}\left[-2 k^{2}+\left(-1+2 \sqrt{1+k^{2}}\right)\left(-1+\sqrt{1+4 k^{2}}\right)\right](-1+r)(1+r)\right\}}{2\left(-3+4 \sqrt{1+k^{2}}-\sqrt{1+4 k^{2}}\right)\left(1+r^{2}\right)}
$$

where

$$
\begin{aligned}
F 1= & {\left[6\left(-1+\sqrt{1+k^{2}}\right)+\left(-5+4 \sqrt{1+k^{2}}-\sqrt{1+4 k^{2}}\right.\right.} \\
& \left.\left.+2 \sqrt{1+k^{2}} \sqrt{1+4 k^{2}}\right) r^{2}-k^{2}\left(3+5 r^{2}\right)\right], \\
F 2= & {\left[-5+4 \sqrt{1+k^{2}}-\sqrt{1+4 k^{2}}+2 \sqrt{1+k^{2}} \sqrt{1+4 k^{2}}-6 r^{2}\right.} \\
+ & \left.6 \sqrt{1+k^{2}} r^{2}-k^{2}\left(5+3 r^{2}\right)\right] .
\end{aligned}
$$

The $\dot{\alpha}=1, n=3$ problem. The variations of the amplitude $A(\tau)$ with the slow time scale appear as inhomogeneous terms in the boundary conditions at order $\alpha=1, n=3$. The governing equations are

$$
\begin{gathered}
i k \tilde{v}_{x}^{l}+\partial_{y} \tilde{v}_{y}^{l}=0, \\
-i k \tilde{p}_{f}^{l}+\left(\partial_{y}^{2}-k^{2}\right) \tilde{v}_{x}^{l}+i k \psi^{l}\left(\partial_{y}^{2} \Psi^{l}\right) \\
=2 i k^{3} \widetilde{\psi}^{* l(1,1)} \widetilde{\psi}^{l(2,2)}+2 i k \widetilde{\psi}^{l(1,1)} \partial_{y}^{2} \widetilde{\psi}^{l(0,2)} \\
+2 i k \widetilde{\psi}^{l(2,2)} \partial_{y}^{2} \widetilde{\psi}^{* l(1,1)}-i k \widetilde{\psi}^{* l(1,1)} \partial_{y}^{2} \widetilde{\psi}^{l(2,2)}, \\
-\partial_{y} \tilde{p}_{f}^{l}+\left(\partial_{y}^{2}-k^{2}\right) \tilde{v}_{y}^{l}+\left(\partial_{y} \Psi^{l}\right)\left(\partial_{y}^{2}-k^{2}\right) \psi^{l}+\left(\partial_{y} \psi^{l}\right)\left(\partial_{y}^{2} \Psi^{l}\right) \\
=-2 k^{2} \widetilde{\psi}^{l(1,1)} \partial_{y} \widetilde{\psi}^{l((0,2)}-4 k^{2} \widetilde{\psi}^{l(2,2)} \partial_{y} \widetilde{\psi}^{* l(1,1)} \\
-k^{2} \widetilde{\psi}^{* l(1,1)} \partial_{y} \widetilde{\psi}^{l(2,2)}+2 \partial_{y} \widetilde{\psi}^{l(1,1)} \partial_{y}^{2} \widetilde{\psi}^{l(0,2)} \\
+2 \partial_{y} \widetilde{\psi}^{l(0,2)} \partial_{y}^{2} \widetilde{\psi}^{l(1,1)}+\partial_{y} \widetilde{\psi}^{l(2,2)} \partial_{y}^{2} \widetilde{\psi}^{* l(1,1)} \\
+\partial_{y} \widetilde{\psi}^{* l(1,1)} \partial_{y}^{2} \widetilde{\psi}^{l(2,2)}, \\
-\widetilde{\psi}^{l(1,3)}-k^{2} \widetilde{\psi}^{l(1,3)}+\partial_{y}^{2} \widetilde{\psi}^{l(1,3)}=0 .
\end{gathered}
$$

The governing equations can be solved to get eigenfunctions that can be written as follows:

$$
\begin{gathered}
\widetilde{\psi}^{a(1,3)}=M_{a 3}^{(1,3)} \exp \left[-\sqrt{1+k^{2}} y\right], \\
\widetilde{\psi}^{b(1,3)}=M_{b 3}^{(1,3)} \exp \left[\sqrt{1+k^{2}} y\right], \\
\tilde{v}_{y}^{a(1,3)}=\left(M_{a 1}^{(1,3)}+M_{a 2}^{(1,3)} y\right) \exp [-k y], \\
\tilde{v}_{y}^{b(1,3)}=\left(M_{b 1}^{(1,3)}+M_{b 2}^{(1,3)} y\right) \exp [k y] .
\end{gathered}
$$

The inhomogeneous boundary conditions in the $(1,3)$ problem are

$$
\begin{gathered}
\tilde{v}_{y}^{a(1,3)}-\tilde{v}_{y}^{b(1,3)}=g_{1}, \\
\tilde{v}_{x}^{a(1,3)}+\left(\partial_{y} \mathbf{v}_{x}^{a}\right) \tilde{u}_{y}^{(1,3)}=g_{2},
\end{gathered}
$$

$$
\begin{gathered}
\tilde{v}_{x}^{b(1,3)}+\left(\partial_{y} \mathbf{v}_{x}^{b}\right) \tilde{u}_{y}^{(1,3)}=g_{3}, \\
\tilde{\tau}_{y y}^{a(1,3)}-\tilde{\tau}_{y y}^{b(1,3)}-\Gamma k^{2} \tilde{u}_{y}^{(1,3)}=g_{4}, \\
\psi^{a(1,3)}+\left(\partial_{y} \Psi^{a}\right) \tilde{u}_{y}^{(1,3)}=g_{5}, \\
\psi^{b(1,3)}+\left(\partial_{y} \Psi^{b}\right) \tilde{u}_{y}^{(1,3)}=g_{6}, \\
\tilde{v}_{y}^{a(1,3)}-s \tilde{u}_{y}^{(1,3)}=g_{7} .
\end{gathered}
$$

The eigenfunctions when substituted in the boundary conditions can then be written in the matrix form as

$$
\mathbf{C} \mid \mathbf{A}=\mathbf{B}
$$

where $\mathbf{C}=\left(c_{i j}\right)$ is the coefficient matrix, vector $\mathbf{A}$ $=\left[M_{a 1}^{(1,3)}, M_{a 2}^{(1,3)}, M_{a 3}^{(1,3)}, M_{b 1}^{(1,3)}, M_{b 2}^{(1,3)}, M_{b 3}^{(1,3)}, \tilde{u}_{y}^{(1,3)}\right] \quad$ while vector $\mathbf{B}=\left[g_{1}, g_{2}, g_{3}, g_{4}, g_{5}, g_{6}, g_{7}\right]$. The time derivative of the amplitude is present in the inhomogeneity $g_{7}$ and the expression for the Landau coefficient is obtained using the solvability condition for the matrix equation. The adjoint problem for Eq. (A65) is constructed by defining the inner product of two vectors $\mathbf{u}$ and $\mathbf{v}$ as

$$
\langle\mathbf{u}, \mathbf{v}\rangle=\sum u_{i}^{\dagger} v_{i}
$$

where $u_{i}^{\dagger}$ is the complex conjugate of $u_{i}$. Using the definition of adjoint we get

$$
\mathbf{C}^{+} \mathbf{A}^{+}=\mathbf{0}
$$

where $\mathbf{A}^{+}=\left[c_{1}, c_{2}, c_{3}, c_{4}, c_{5}, c_{6}, c_{7}\right]$ is the nontrivial adjoint solution for the homogeneous adjoint problem, and $\mathbf{C}^{+}$ $=\left(c_{j i}^{+}\right)$is the adjoint of the matrix $\mathbf{C}$. The Landau equation is then obtained using the Fredholms solvability condition by setting the solution of the adjoint problem orthogonal to the inhomogeneities,

$$
\mathbf{A}^{+} \mathbf{B}=\mathbf{0} .
$$

\section{APPENDIX B}

Here we give the numerical procedure for calculating eigenfunctions in the case of the PB model. Details are given for the linear problem and similar procedure holds for all the other orders of nonlinearity. The governing equations to be solved are

$$
\begin{gathered}
\left(\partial_{y}^{2}-k^{2}\right) \widetilde{\psi}^{l(1,1)}=\cosh \left[\Psi_{m}^{a}\right] \widetilde{\psi}^{l(1,1)}, \\
\left(\partial_{y}^{2}-k^{2}\right)^{2} \widetilde{v}_{y}^{l(1,1)}=0 .
\end{gathered}
$$


The governing equation for the $y$ velocity is decoupled and two decaying eigenfunctions admitted are given by

$$
\tilde{v}_{y}=e^{-k y} \quad \text { and } y e^{-k y} .
$$

For Eq. (B1) however analytical solutions are difficult. The equation is solved numerically by making a coordinate transformation $y=e^{-y}$ so that the domain of integration is transformed from $y=0$ and $y=\infty$ to $y=1$ and $y=0$. A similar transformation can be made for the bottom fluid namely, $y$ $=e^{y}$ and the integration limits in this case get transformed from $y=-\infty$ and $y=0$ to $y=0$ and $y=1$. The transformed equation then becomes

$$
\left(y^{2} \partial_{y}^{2}+y \partial_{y}-k^{2}\right) \widetilde{\psi}^{l(1,1)}=\cosh \left[\Psi_{m}^{a}\right] \widetilde{\psi}^{l(1,1)} .
$$

To integrate Eq. (B4) numerically we need two boundary conditions at $y=0$. The equations are however singular at $y=0$. The integration is therefore proceeded from a small value of $y=y_{0}$ and is verified to be independent of the choice of $y_{0}$. The boundary conditions are the solutions for the $\mathrm{DH}$ model in the $y=0$ limit. The decaying eigenfunction for the DH model is given by (A7), so that the boundary condition at $y=y_{o}$ becomes

$$
\begin{gathered}
\left.\widetilde{\psi}^{l(1,1)}\right|_{y=y_{0}}=y_{0}^{\sqrt{\left(k^{2}+1\right)}}, \\
\left.\partial_{y} \widetilde{\psi}^{l(1,1)}\right|_{y=y_{0}}=\sqrt{\left(k^{2}+1\right)} y_{0}^{\sqrt{\left(k^{2}+1\right)-1}} .
\end{gathered}
$$

The numerical procedure is same for all other orders. For the adjoint problem, the homogeneous solution is obtained by the above procedure. The nonhomogeneous solution is then obtained using homogeneous boundary conditions at $y=y_{0}$.
[1] A. Voight and E. Donath, in Biophysics of the Cell Surface, edited by R. Glaser and D. Gingell (Springer-Verlag, Berlin, 1990).

[2] E. W. Kaler, A. K. Murthy, B. E. Rodriguez, and J. A. N. Zasadzinski, Science 245, 1371 (1989).

[3] M. D. Houslay and K. K. Staney, Dynamics of Biological Membranes (Wiley, New York, 1982).

[4] S. A. Safran, P. A. Pincus, D. Andelman, and F. C. MacKintosh, Phys. Rev. A 43, 1071 (1991).

[5] P. B. S. Kumar and M. Rao, Phys. Rev. Lett. 80, 2489 (1998).
[6] V. Kumaran, Phys. Rev. E 64, 011911 (2001).

[7] M. Winterhalter and W. Helfrich, J. Phys. Chem. 92, 6865 (1988).

[8] H. N. W. Lekkerkerker, Physica A 167, 384 (1990).

[9] J. Oberdisse and G. Porte, Phys. Rev. E 56, 1965 (1997).

[10] J. Oberdisse, Eur. Phys. J. B 3, 463 (1998).

[11] V. Kumaran, J. Chem. Phys. 99, 5490 (1993).

[12] V. Kumaran, Phys. Rev. Lett. 85, 4996 (2000); Phys. Rev. E 64, 051922 (2001). 